\title{
Spontaneous edge order and geometric aspects of two-dimensional Potts models
}

\author{
Youjin Deng ${ }^{1, *}$ and Henk W. J. Blöte ${ }^{1,2}$ \\ ${ }^{1}$ Faculty of Applied Sciences, Delft University of Technology, P.O. Box 5046, 2600 GA Delft, The Netherlands \\ ${ }^{2}$ Lorentz, Institute, Leiden University, P.O. Box 9506, 2300 RA Leiden, The Netherlands
}

(Received 3 June 2004; published 30 September 2004)

\begin{abstract}
Using suitable Monte Carlo methods and finite-size scaling, we investigate critical and tricritical surface phenomena of two-dimensional Potts models. For the critical two- and three-state models, we determine a surface scaling dimension describing percolation properties of the so-called Potts clusters near the edges. On this basis, we propose an exact expression describing this exponent for the whole critical branch. For tricritical Potts models we find that varying the surface coupling constant or the surface magnetic field can induce a continuous phase transition. At bulk tricriticality and sufficiently strong surface couplings, spontaneous onedimensional order occurs on the edges. We determine several critical exponents describing these edge transitions. On the basis of these results and conformal field theory, we conjecture exact expressions for these exponents.
\end{abstract}

DOI: 10.1103/PhysRevE.70.035107

PACS number(s): 05.50.+q, 64.60.Cn, 64.60.Fr, 75.10.Hk

In two dimensions, the nature of bulk critical singularities is now well established for the $q$-state Potts model [1-3]. Nevertheless, a number of interesting questions remain unanswered. For instance, exact values have not been obtained for some geometric critical exponents [4]; and it was recently reported [5] that, for the tricritical branch, the percolation threshold of the so-called geometric clusters does not coincide with the well-known Kasteleyn-Fortuin (KF) clusters [6]. Surface critical behavior of the Potts model on the half plane also remains largely unexplored. In this context, we briefly review the three-dimensional Ising model in the half space [7,8]. Depending on the surface coupling constant $K_{s}$, the transition at $K=K_{c}$ may occur in the absence or presence of a spontaneously ordered surface: the "ordinary" or the "extraordinary" transition, respectively. For disordered bulk $K<K_{c}$, there is a critical line of "surface" transitions terminating at $K=K_{c}$ in a multicritical point, the so-called "special" transition.

In two dimensions, the "surfaces" are just onedimensional edges, and thus no surface transition can exist in systems with short-range interactions. It may then seem natural to expect that special and extraordinary transitions do not occur either, i.e., that spontaneous edge order is absent. Whereas this expectation has been rigorously confirmed for the critical Ising model $[9,10]$, in this work we show that it does not hold in general.

Just as the Potts bulk critical singularities are geometrically represented by the fractal dimension $X_{h}$ of the aforementioned KF clusters [6], the surface critical properties are governed by a surface exponent $y_{h s}^{(\mathrm{o})}$, where (o) indicates the ordinary transition. For general critical $q$-state Potts models, Cardy [11] employed boundary conformal field theory to express this exponent in terms of the bulk thermal one $y_{t}$ as

\footnotetext{
*Present address: Laboratory for Material Science, Delft University of Technology, Rotterdamseweg 137, 2628 AL Delft, The Netherlands.
}

$$
y_{h s}^{(\mathrm{o})}=2-3 /\left(3-y_{t}\right)=2-g / 2,
$$

where the Coulomb gas coupling constant $g$ is related to $q$ as $q=2+2 \cos (g \pi / 2)[2,3]$. Remarkably, $y_{h s}$ in Eq. (1) is a decreasing function of $g$. The surface magnetic field $H_{s}$ is then marginal $\left(y_{h s}^{(\mathrm{o})}=0\right)$ for $q=4$. Moreover, generalization of Eq. (1) would yield that $H_{s}$ is irrelevant for the tricritical Potts model $(g>4)$. However, since the value of $g$ reflects the strength of critical fluctuations, one might instead expect that the effect of $H_{s}$ is stronger on the tricritical branch than on the critical one. Further exploration of this paradox seems warranted.

Accordingly, the present paper investigates the nearestneighbor Potts model on an open cylinder, i.e., a $L \times L$ square lattice with periodic and open boundary conditions in the $x$ and $y$ directions, respectively. In order to allow for tricritical points, we include vacancies in the Hamiltonian. Thus, for the Ising case $q=2$, we take spin- $1\left(s_{i}= \pm 1,0\right)$ variables,

$$
\begin{aligned}
\mathcal{H} / k_{\mathrm{B}} T= & -K \sum_{\langle i j\rangle}{ }^{(\mathrm{b})} s_{i} s_{j}+D \sum_{k}{ }^{(\mathrm{b}, \mathrm{s})} s_{k}^{2}-K_{s} \sum_{\langle l m\rangle}{ }^{(\mathrm{s})} s_{l} s_{m} \\
& -H_{s} \sum_{n}{ }^{(\mathrm{s})} s_{n}
\end{aligned}
$$

where the first sum accounts for the bulk and the last two sums for the open edges. The abundance of vacancies $\left(s_{i}\right.$ $=0)$ is controlled by the chemical potential $D, H_{s}$ is the surface magnetic field, and $K$ and $K_{s}$ represent the bulk and surface couplings, respectively. For $D \rightarrow-\infty$, the model (2) reduces to the $s=1 / 2$ Ising model. The Wolff algorithm [12] was used to simulate its bulk critical point $K_{s}=K=K_{c}=\ln (1$ $+\sqrt{2}) / 2, H_{s}=0$, for 18 system sizes in the range $8 \leqslant L$ $\leqslant 600$. In addition to KF clusters, we also investigate Potts clusters [13], which connect all nearest-neighbor spins in the same state. The sampling procedure includes the probability $C(L / 2)$ that two surface points at a distance $L / 2$ are in the same Potts cluster. The data for $C$, shown in Fig. 1, were fitted by 


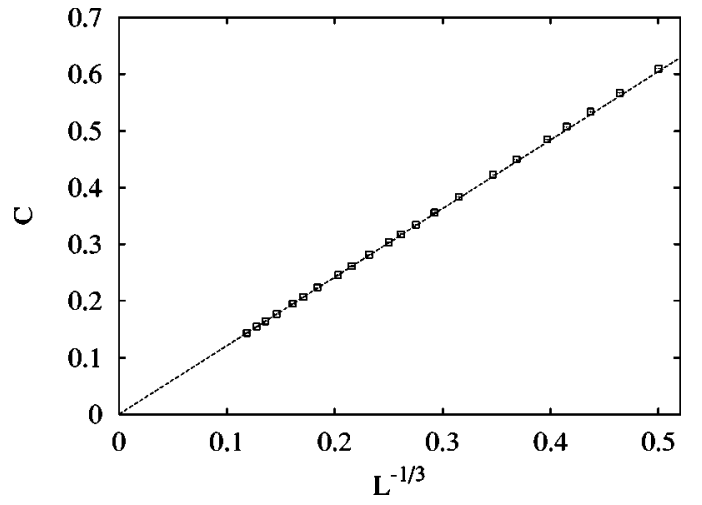

FIG. 1. Surface correlation function $C$ for the critical Ising model vs $L^{-1 / 3}$ for system sizes in the range $8 \leqslant L \leqslant 600$. The statistical errors are much smaller than the size of the data points.

$$
C=L^{-2 X_{p s}^{(\mathrm{o})}}\left(a_{0}+a_{1} L^{y_{1}}+a_{2} L^{y_{2}}+a_{3} L^{y_{3}}\right) .
$$

The leading correction exponent $y_{1}$ was taken as the tricritical $q=1$ red-bond exponent $y_{r}=-5 / 8[5,13]$, governing the renormalization of the bond probability. With $y_{2}=-1$ and $y_{3}=-2$, we obtain $X_{p s}^{(\mathrm{o})}=0.16663(8) \approx 1 / 6$, apparently distinct from $X_{h s}^{(\mathrm{o})}=1 / 2$.

Next, we consider the dilute three-state Potts model,

$$
\begin{aligned}
\mathcal{H} / k_{\mathrm{B}} T= & -K \sum_{\langle i j\rangle}{ }^{(\mathrm{b})} \delta_{\sigma_{i}, \sigma_{j}}\left(1-\delta_{\sigma_{i}, 0}\right)-D \sum_{k}{ }^{(\mathrm{b}, \mathrm{s})} \delta_{\sigma_{i}, 0} \\
& -K_{s} \sum_{\langle l m\rangle}{ }^{(\mathrm{s})} \delta_{\sigma_{l}, \sigma_{m}}\left(1-\delta_{\sigma_{m}, 0}\right)-H_{s} \sum_{n}{ }^{(\mathrm{s})} \delta_{\sigma_{n}, 1},
\end{aligned}
$$

where $\sigma=0$ stands for a vacancy and $\sigma=1,2$, and 3 for a Potts spin. The simulations were performed for $D \rightarrow-\infty$ at criticality $K_{s}=K=K_{c}=\ln (1+\sqrt{3})$ and $H_{s}=0$ [1], with $8 \leqslant L$ $\leqslant 600$. The data for $C$ were fitted by Eq. (3) with $y_{1}$ $=-23 / 60$, the red-bond exponent of the tricritical $q=2$ $+2 \cos (2 \pi / 5)$ Potts model [5,13]. The fit yields $X_{p s}^{(\mathrm{o})}$ $=0.3999(1) \approx 2 / 5$. It is obvious that $X_{p s}^{(\mathrm{o})}=0$ for the percolation model $(q \rightarrow 1)$, since all lattice sites are within a single Potts cluster. In contrast, the $q=4$ case is marginal $\left(y_{r}=0\right)$, so that we expect $X_{p s}^{(\mathrm{o})}=X_{h s}^{(\mathrm{o})}=1$.

Conformal field theory captures a number of critical indices of two-dimensional Potts models by the Kac formula $[11,14,15]$,

$$
\Delta_{p, q}=\frac{[(m+1) p-m q]^{2}-1}{4 m(m+1)},
$$

where $m=g /(4-g)$ and $4 /(g-4)$ for critical and tricritical branches, respectively. For instance, $X_{h s}^{(\mathrm{o})}$ in Eq. (1) can be interpreted as $\Delta_{1,3}$ in Eq. (5). We propose $X_{p s}^{(\mathrm{o})}=\Delta_{3,5}$, i.e.,

$$
X_{p s}^{(\mathrm{o})}=\frac{(m-1)(m-2)}{m(m+1)}=\frac{(3 g-8)(g-2)}{2 g},
$$

in fine agreement with the aforementioned values for $q=1,2$, 3 , and 4 .

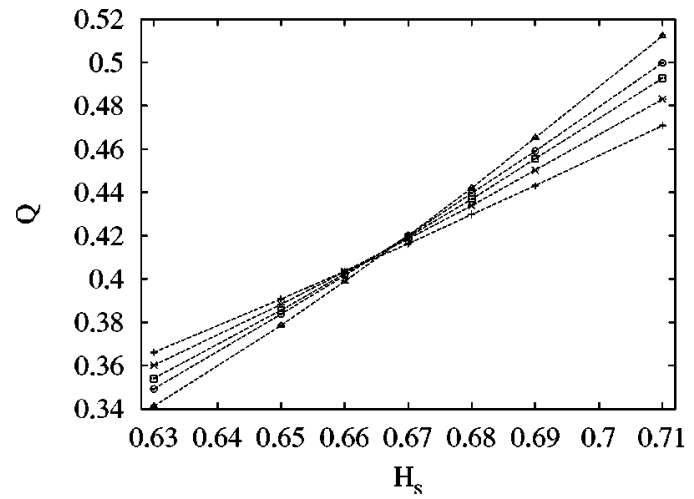

FIG. 2. Dimensionless surface ratio $Q$ for the tricritical Ising model vs surface magnetic field $H_{s}$. The data points $+, \times, \square, \bigcirc$, and $\triangle$, represent $L=8,12,16,20$, and 28 , respectively.

Boundary conformal field theory has already been applied to the tricritical Ising model [15-18], which corresponds with an integrable scattering theory of massive kinks and preserves superconformal symmetry. By means of factorizable $S$ matrix, fusion rules, and symmetry arguments, various boundary operators and the corresponding renormalization flows were conjectured [16]. A physical interpretation of these boundary phenomena was then provided by Affleck [17], which indicates the possible existence of spontaneous magnetization on the edges. By means of a sparse transfermatrix technique, we [19] located the tricritical point of the Ising model (2) at $K_{t c}=1.6431758(1)$ and $D_{t c}$ $=3.2301797(2)$. The surface coupling was set at $K_{s}=K_{t c}$, and Eq. (2) for $H_{s} \neq 0$ was simulated using Metropolis sweeps, with $8 \leqslant L \leqslant 48$. We sampled the surface magnetization density $m_{s}$ and obtained the dimensionless surface ratio $Q=\left(\left\langle m_{s}^{2}\right\rangle-\left\langle m_{s}\right\rangle^{2}\right)^{2} /\left\langle\left(m_{s}-\left\langle m_{s}\right\rangle\right)^{4}\right\rangle$. For small $H_{s}$, the edges appear to be dominated by vacancies: $H_{s}$ is indeed irrelevant as predicted by Eq. (1). For $H_{s} \gg 0$, however, the edges are mainly occupied by plus spins $s=+1$, and a continuous transition separates these two phases. This follows from the clear intersections in the $Q$ versus $H_{s}$ data, partly shown in Fig. 2. The data were fitted by

$$
\begin{aligned}
Q\left(H_{s}, L\right)= & Q_{0}+\sum_{k=1}^{4} q_{k}\left[\left(H_{s}-H_{s c}\right) L^{y_{h s}^{(\mathrm{f})}}\right]^{k}+\sum_{j=1}^{3} b_{j} L^{y_{j}} \\
& +c\left(H_{s}-H_{s c}\right) L^{y_{1}+y_{h s}^{(\mathrm{f})}}
\end{aligned}
$$

where the superscript (f) represents a nonzero field $H_{s}$. The term with amplitude $c$ accounts for the bilinear effect of the relevant and the irrelevant field. We set $y_{j}=-j$ and obtained a satisfactory fit, thus confirming the continuous transition induced by $H_{s}$. We find $Q_{0}=0.4419(15), H_{s c}=0.6772(10)$, and $y_{h s}^{(\mathrm{f})}=0.405(10) \approx 2 / 5$. We also simulated the $q=3$ Potts model (4) at tricriticality $K_{t c}=1.649913(5)$ and $D_{t c}$ $=3.152$ 173(10) [19], using a combination of Metropolis and Wolff steps, where the latter flip between states $\sigma=2$ and $\sigma$ $=3$ only. The edge order parameter $m_{s}$ was chosen as the density of edge spins $\rho_{1}$ in state $\sigma=1$. Equation (7) was fitted to the data for $Q$ in the range $8 \leqslant L \leqslant 40$, and we obtain $Q_{0}$ 


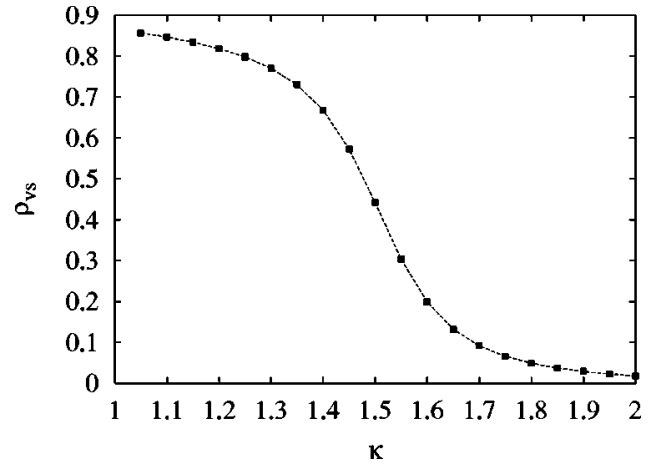

FIG. 3. The edge vacancy density $\rho_{v s}$ of the tricritical Ising model vs the enhancement $\kappa$. The system size is $L=15$.

$=0.462(3), H_{t c}=0.5710(15)$, and $y_{h s}^{(\mathrm{f})}=0.278(8)$.

In accordance with these numerical results for $y_{h s}^{(\mathrm{f})}$, we propose $X_{h s}^{(\mathrm{f})}=\Delta_{1,3}$ in Eq. (5), which also relates to the subleading thermal dimension as $2 \Delta_{1,3}=X_{t 2}$. Then, one has

$$
X_{h s}^{(\mathrm{f})}=8 / g-1,
$$

which, we conjecture, holds for the whole tricritical branch.

Next, we simulated the tricritical Ising model in the range $8 \leqslant L \leqslant 120$ at $H_{s}=0$. At bulk tricriticality, the vacancies dominate on the edges for $K_{s} \lesssim K_{t c}$, but are squeezed out by a sufficient enhancement of $K_{s}$, as shown in Fig. 3. Further, the clean intersection of the $Q$ data in Fig. 4 indicates the existence of a second-order phase transition. Adopting the language of the three-dimensional case, we refer to the edge transition in Fig. 4 as the special transition. The data for $Q$ were fitted by Eq. (7) with $y_{h s}^{(\mathrm{f})}$ replaced by $y_{\kappa}^{(\mathrm{s})}$ and $H_{s}$ by $\kappa=K_{s} / K_{t c}$; we obtain $Q_{0}=0.744(2), \kappa_{c}=1.5662(3)$, and $y_{\kappa}^{(\mathrm{s})}$ $=0.398(4)$. For the tricritical three-state Potts model, the edge order parameter was defined as $m_{s}^{2}=\Sigma_{i \neq j}\left(\rho_{i}-\rho_{j}\right)^{2} / 2$, with $\rho_{i}$ the density of edge spins in state $i$. For system sizes in the range $8 \leqslant L \leqslant 64$, the fit to the data for $Q$ yields $Q_{0}$ $=0.941(2), \kappa_{c}=1.702(2)$, and $y_{\kappa}^{(\mathrm{s})}=0.282(5)$.

Since our results for $y_{h s}^{(\mathrm{f})}$ and $y_{\kappa}^{(\mathrm{s})}$ coincide, and $H_{s}$ and $K_{s}$ play a similar role in the sense that they suppress the edge vacancies, we assume that $y_{\kappa}^{(\mathrm{s})}=1-X_{h s}^{(\mathrm{f})}$ as gained in Eq. (8). Some further insight can be gleaned from the equivalence of

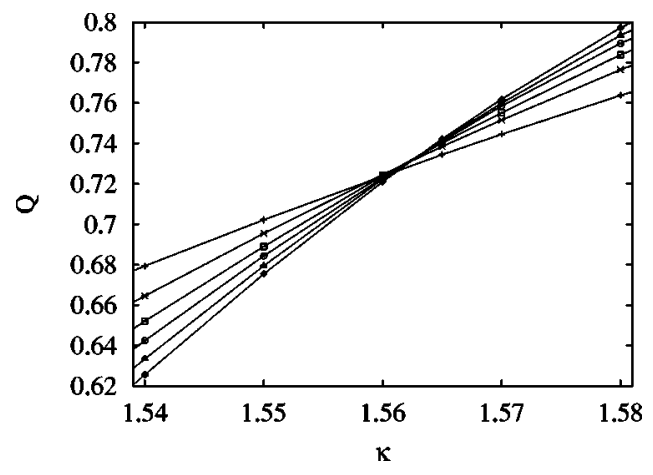

FIG. 4. The ratio $Q$ for the tricritical Ising model vs $\kappa=K_{s} / K_{t c}$. The data points $+, \times, \square, \bigcirc, \triangle$, and $\diamond$ represent $L=8,16,24,32$, 40 , and 48 , respectively.

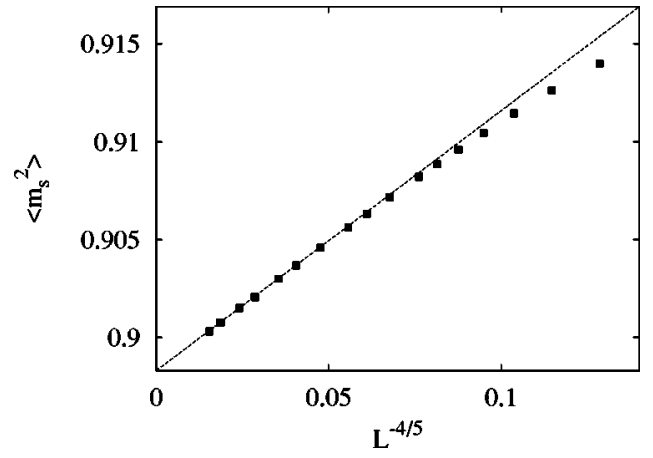

FIG. 5. The squared spontaneous edge magnetization $\left\langle m_{s}^{2}\right\rangle$ at the extraordinary transition of the tricritical Ising model vs $L^{-4 / 5}$, for system sizes $13 \leqslant L \leqslant 185$. The surface coupling is given by $\kappa$ $=1.805$.

the tricritical $q=1$ Potts and the critical Ising model [2]. The surface coupling $K_{s}$ in the former plays a role of the surface magnetic field $H_{s}$ in the latter. Thus, Eq. (1) yields $y_{\kappa}^{(\mathrm{s})}$ $=1 / 2$ for the tricritical $q=1$ Potts model, in agreement with Eq. (8). Let us further conjecture that the effect of $K_{s}$ on a tricritical Potts model is equivalent to that of $H_{s}$ on a critical system, the two models being related by $g g^{\prime}=16$. We note that it has been argued earlier that, for such a pair of models [20], the fractal dimension $X_{p}$ of the critical model is equal to $X_{h}$ of the tricritical one [5]. Indeed, the substitution $g$ $=16 / g^{\prime}$ in Eq. (1) reproduces Eq. (8). Although the conformal anomalies for $g$ and $g^{\prime}$ coincide, the physical picture of the equivalence conjectured above is not obvious for $q \neq 1$.

At the special transition $\kappa=\kappa_{c}$, we have also analyzed the surface susceptibility $\chi_{1}=L\left\langle m_{s}^{2}\right\rangle$, and obtain $X_{h s}^{(\mathrm{s})}(q=2)$ $=0.09(1)$ and $X_{h s}^{(\mathrm{s})}(q=3)=0.133(15)$. Accordingly, we propose $X_{h s}^{(\mathrm{s})}=\Delta_{1,2}$ in Eq. (5), which relates to the leading bulk thermal dimension via $X_{t 1}=2 \Delta_{1,2}$. Then, we conjecture for the whole tricritical branch,

$$
X_{h s}^{(\mathrm{s})}=(6-g) / 2 g .
$$

For $\kappa>\kappa_{c}$, a spontaneous surface magnetization occurs, and the surface transition is first order at $K=K_{c}$, to which we refer as the extraordinary transition. The data for $\left\langle m_{s}^{2}\right\rangle$ of the tricritical Ising model, as shown in Fig. 5, were fitted by

$$
\left\langle m_{s}^{2}\right\rangle=a+L^{-2 X_{h s}^{(\mathrm{e})}}\left(b_{0}+b_{1} / L+b_{2} / L^{2}+b_{3} / L^{3}\right),
$$

which yields $a=0.89823(3)$ and $X_{h s}^{(\mathrm{e})}=0.3987(15) \approx 2 / 5$, indicating that the edges are still critical, even though the edge transition is first order. This is further shown by the ratio $Q_{b}$ in Fig. 6, where the magnetization density for $Q_{b}$ was only sampled on the middle line of the system, i.e., $y=(L+1) / 2$. The clean intersection of the $Q_{b}$ data in Fig. 6 indicates the existence of a fixed point near $\kappa=1.805$; further, the decay of the slopes, as $L$ increases, means that this fixed point is stable in the $\kappa$ direction. Thus, although most conjectures in Refs. $[16,17]$ are consistent with our numerical simulations, Fig. 6 suggests that the renormalization flow in Fig. 1 in Ref. [17] might not be complete.

As an explanation of the paradox mentioned after Eq. (1), we note that the critical state on the edges is affected by the 


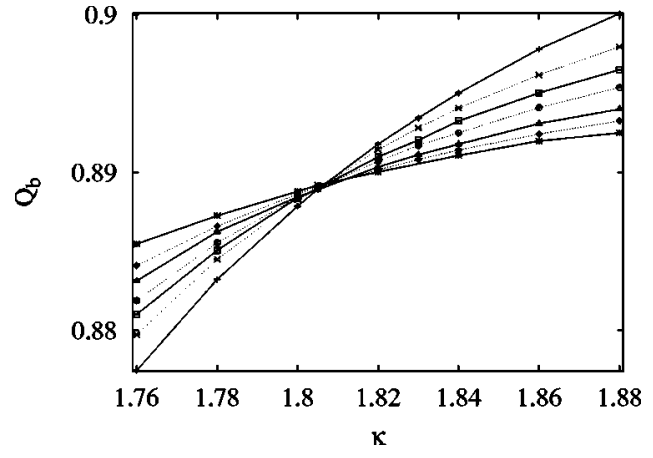

FIG. 6. The ratio $Q_{b}$ for the tricritical Ising model vs $\kappa$ $=K_{s} / K_{t c}$. The data points $+, \times, \square, \bigcirc, \triangle, \diamond$, and $*$ represent $L$ $=7,9,11,13,17,21$, and 29 , respectively.

geometric effect of the missing bonds. The magnitude of this effect relates to the bulk exponent $y_{t}$. For $g<4, y_{t}$ is still relatively small and the edges maintain strong critical correlations. However, on the tricritical branch $(g>4)$, the geometric effect is so large that vacancies dominate and the edge correlations decrease to the extent that $H_{s}$ becomes irrelevant. This effect can be compensated by surface parameters, such as $H_{s}$ and $K_{s}$, which then induce various edge transitions.
Some final remarks follow. First, at the bulk tricriticality, the decay of surface effects into the bulk is long ranged and governed by bulk critical exponents. Second, the correspondence between the bulk dimension $X_{p}$ of critical Potts clusters and $X_{h}$ of a tricritical system [5] suggests that also $X_{p s}^{(\mathrm{o})}$ in Eq. (6) relates to some tricritical exponent. Third, it is known [21] that the surface thermal exponent is $y_{\kappa}=-1$ for ordinary transitions; while Eq. (8) yields a marginal exponent $y_{\kappa}=1$ $-x_{\kappa}=0$ for the $q=4$ Potts model. There are also two different predictions for the surface magnetic dimensions in this case: $X_{h s}^{(\mathrm{o})}=1$ by Eq. (1) and $X_{h s}^{(\mathrm{s})}=1 / 4$ by Eq. (9). This indicates the existence of a special transition for the $q=4$ Potts model.

In conclusion, even in two-dimensional systems with short-range interactions only, rich critical surface phenomena occur. This is possible only because the edge spins are correlated through the critical bulk.

We are indebted to J. L. Cardy, T. Halpin-Healy, J. R. Heringa, and B. Nienhuis for valuable discussions and comments. This research was supported by the Dutch FOM Foundation ("Stichting voor Fundamenteel Onderzoek der Materie"), which was financially supported by the NWO ("Nederlandse Organisatie voor Wetenschappelijk Onderzoek").
[1] F. Y. Wu, Rev. Mod. Phys. 54, 235 (1982).

[2] B. Nienhuis, A. N. Berker, E. K. Riedel, and M. Schick, Phys. Rev. Lett. 43737 (1979).

[3] B. Nienhuis, Phase Transitions and Critical Phenomena, edited by C. Domb and J. L. Lebowitz (Academic, London, 1987).

[4] H. J. Herrmann and H. E. Stanley, Phys. Rev. Lett. 53, 1121 (1984); P. Grassberger, Physica A 262, 251 (1999); Y. Deng, H. W. J. Blöte, and B. Nienhuis, Phys. Rev. E 69, 026114 (2004).

[5] Y. Deng, H. W. J. Blöte, and B. Nienhuis, Phys. Rev. E 69, 026123 (2004).

[6] P. W. Kasteleyn and C. M. Fortuin, J. Phys. Soc. Jpn. 46 (Suppl.), 11 (1969); C. M. Fortuin and P. W. Kasteleyn, Physica (Amsterdam) 57, 536 (1972).

[7] K. Binder, Phase Transitions and Critical Phenomena, edited by C. Domb and J. L. Lebowitz (Academic, London, 1987), Vol. 8, p. 1; H. W. Diehl, Phase Transitions and Critical Phenomena, Vol. 10, p. 76.

[8] D. P. Landau and K. Binder, Phys. Rev. B 41, 4633 (1990).

[9] B. M. McCoy and T. T. Wu, Phys. Rev. 162, 436 (1967).

[10] H. Au-Yang and M. E. Fisher, Phys. Rev. B 21, 3956 (1980).
[11] J. L. Cardy, Nucl. Phys. B 240 [FS12], 514 (1984); ibid. 324, 581 (1989).

[12] U. Wolff, Phys. Rev. Lett. 62, 361 (1989).

[13] A. L. Stella and C. Vanderzande, Phys. Rev. Lett. 62, 1067 (1989); B. Duplantier and H. Saleur, ibid. 63, 2536 (1989); H. W. J. Blöte, Y. M. M. Knops, and B. Nienhuis, ibid. 68, 3440 (1992).

[14] A. A. Belavin, A. M. Polyakov, and A. B. Zamolodchikov, J. Stat. Phys. 34, 763 (1984); D. Friedan, Z. Qiu, and S. Shenker, Phys. Rev. Lett. 52, 1575 (1984).

[15] J. L. Cardy, Phase Transitions and Critical Phenomena, edited by C. Domb and J. L. Lebowitz (Academic, London, 1987), Vol. 11, p. 55.

[16] L. Chim, Int. J. Mod. Phys. A 11, 4491 (1996).

[17] I. Affleck, J. Phys. A 33, 6473 (2000).

[18] A. De Martino and M. Moriconi, Nucl. Phys. B 528, 577 (1998); G. Feverati, P. A. Pearce, and F. Ravanini, Phys. Lett. B 534, 216 (2002); Nucl. Phys. B 675, 469 (2003); R. I. Nepomechie, Int. J. Mod. Phys. A 17, 3809 (2002).

[19] X. F. Qian, H. W. J. Blöte, and Y. Deng (unpublished).

[20] B. Duplantier, Phys. Rev. Lett. 84, 1363 (2000).

[21] T. W. Burkhardt and J. L. Cardy, J. Phys. A 20, L233 (1987). 